\title{
IL SUCCESSO ITALIANO DEL PROFESSOR JERZY TOPOLSKI
}

\section{Stanisław SIERPOWSKI}

La produzione del prof. Topolski accessibile in lingua italiana è la più ampia fra quella degli storici o più in generale degli umanisti polacchi del ventesimo secolo che non abbiano risieduto per lunghi periodi in Italia. Questa osservazione è d'obbligo, poiché non si può non menzionare l'attività scientifica e pubblicistica del professor Bronisław Biliński, che per quasi tre decadi ha diretto la sede dell'Accademia Polacca delle Scienze nella Città Eterna (vedi sotto). Sembra tuttavia che i libri di Jerzy Topolski suscitassero vivo interesse, discussioni e polemiche. Non trovo alcuno studioso mio coetaneo presente con traduzioni in ambito italofono, i cui lavori si siano stabilmente inseriti nella storiografia italiana, incontrando un costante interesse. Ciò era favorito dalla grande mobilità sia sul territorio nazionale, sia all'estero, nonché dalla risposta positiva qualora fosse invitato a partecipare a seminari o conferenze, delle quali del resto non era entusiasta.

Fra i numerosi talenti che inserivano il prof. Topolski nella schiera dei geni vi era la facilità con cui si appropriava di altre lingue. È difficile stabilire quando si sia trovato nell'ambito italofono. Nei numerosi questionari personali che accompagnarono la carriera del prof. Topolski e in particolare fra l'ampia documentazione relativa al conferimento del titolo di professore, affermava di avere un'ottima conoscenza della lingua francese. Fra le lingue a lui note menzionava inoltre l'inglese, il russo e il tedesco. Nella recensione preparata nell'ambito della procedura di riconoscimento della cattedra intorno alla metà del 1966 il professor Henryk Lowmiański scrisse: "Il candidato mostra un'ampia conoscenza della letteratura inglese, francese, tedesca e italiana e anche di Paesi slavi, di cui seleziona i titoli utili a illustrare la sua fondamentale concezione"'. La segnalazione della conoscenza della lingua italiana presente in questa valutazione era sicuramente

AUAM, Sprawa unadzwyczajnienia prof. Jerzego Topolskiego, sygn. 69/9, p. 3. 
dovuta alla presenza nella bibliografia di Jerzy Topolski di una recensione del libro di Domenico Sella Commerci e industrie a Venezia nel secolo XVII, pubblicata nel 1965 sulla rivista "Roczniki Dziejów Społecznych i Gospodarczych" (Annali di Storia Sociale ed Economica). Si trattava dell'unico testo relativo alla storiografia italiana nell'ambito dell'allora molto intensa attività di recensione, che emergeva anzitutto nelle colonne dei già menzionati "Roczniki"2.

Importante per la presenza di Jerzy Topolski nella vita scientifica internazionale fu l'elezione nel 1970 a membro del Direttivo Internazionale della Società di Storia Economica. Nuove prospettive erano aperte dalla partecipazione alle conferenze organizzate dall'Istituto Internazionale di Storia Economica Francesco Datini di Prato ${ }^{3}$. La svolta si ebbe tuttavia con la traduzione di una delle opere più note al mondo di Jerzy Topolski, Metodologia historii, del 1968. L'edizione italiana era basata sulla seconda edizione polacca del 1973. La traduzione, realizzata dal professor Kazimierz Lewański ${ }^{4}$ e pubblicata a Bologna nel 1975 con il titolo di Metodologia della ricerca storica. Nuovi principi di metodologia storica, fu in assoluto la prima traduzione in una lingua straniera. La traduzione italiana precedette dunque quella inglese del 1976, pubblicata del resto grazie all'impegno e con il marchio dell'Editrice Nazionale Scientifica (Polskie Wydawnictwo Naukowe), quella spagnola del 1981 (la seconda edizione uscì nel 1985) o quella romena del 1987. Va anche osservato che la pubblicazione di questo ampio, rappresentativo lavoro, non fu preceduta da altri testi di Jerzy Topolski in italiano.

Non vi è dubbio che la Metodologia abbia aperto a Jerzy Topolski le porte delle università italiane. Come principale ospite partecipò nel maggio 1976 ai seminari presso le università di Bologna e Milano. All'Università di Sassari si svolse un simposio di tre giorni dedicato esclusivamente alla discussione metodologica. Il professor Sandro Schipani nell'invito del 1 febbraio 1976 scrisse: „Riteniamo che il suo contributo al nostro seminario sia

2 A meno che siano stati tralasciati titoli della bibliografia successiva al 1987, per gli anni 1951-1987 la bibliografia è stata compilata da Maria Danuta Topolska. Vd.: Jerzy Topolski - o nowy ksztatt nauki historycznej, red. M. Drozdowski, Warszawa-Poznań 1988, pp. 66-134. Per le integrazioni vd. eadem, “Studia Historiae Oeconomiae", t. 23 (1998). Per gli anni 1987-1998 e 1998-2010 vd.: Oblicza przesztości, p. 89 e segg.

3 Vd. S. Bielański, Dwadzieścia lat działalności Istituto "Francesco Datini" w Prato, "Historyka", T. XX, 1990.

4 Nella bibliografia dei lavori di Jerzy Topolski sono stati omessi i nomi dei traduttori. Per questo manca il riferimento a Ryszard Kazimierz Lewański (1918-1996), creatore della traduzione in italiano. Si trattava di un personaggio non convenzionale e di grandi meriti per la polonistica e le ricerche slavistiche in Italia. Soldato del Secondo Corpo Polacco, docente nelle scuole americane, negli anni Sessanta creò la polonistica presso l'Università di Pisa e successivamente divenne professore associato di lingua e letteratura polacca all'Università di Udine. Nel 1973 si adoperò per la ricostituzione dell'Accademia Adam Mickiewicz di Bologna, la cui attività era stata interrotta nel 1926, coinvolgendovi anche il sottoscritto. Era considerato un uomo-istituzione. Soprattutto in Italia settentrionale svolgeva un ruolo analogo a Bronisław Biliński nella capitale italiana. Va osservato che nella produzione scientifica del prof. Lewański, concentrata sul XVI secolo e sulla biografistica, mancano titoli dedicati alla metodologia. Anche il professor Andrzej Litwornia, riassumendo le realizzazioni del Lewański (Pamiętnik Literacki LXXXVIII, 1997, z. 4, pp. 229-234), ne ha tralasciato i meriti nel campo della traduzione. 
particolarmente importante. La recente pubblicazione in Italia del suo volume Metodologia della ricerca storica accresce il nostro desiderio di una collaborazione con Lei" ${ }^{5}$.

Di passaggio vale la pena di osservare che Jerzy Topolski come conferenziere nelle istituzioni estere si serviva soprattutto del francese e dell'inglese. Abbastanza rapidamente acquisì anche l'italiano parlato, del quale si serviva - come ho avuto modo di constatare intorno alla metà degli anni Ottanta - con competenza e scioltezza. Lo ritroviamo addirittura nel ruolo di traduttore del non facile testo del prof. Righini sull'immaginario dei Romani intorno alle Indie nei primi due secoli della nostra era ${ }^{6}$. Se negli incontri e nelle lezioni accademiche si sforzava di parlare in italiano, affidava i testi ad abili traduttori, con i quali restava costantemente in contatto, dimostrando nei loro confronti grande stima, serietà e cordialità. Era il caso di un ciclo di lezioni tenute presso l'Università di Perugia nel 1984. Fin dalla primavera di quell'anno il prof. Topolski conduceva trattative con il Dipartimento di Storia relativamente all'avvio di una collaborazione. Controparte italiana era il professor Ettore Deodato, il quale in una lettera rivolta al sottoscritto informava che con il prof. Topolski stavano analizzando tutte le possibilità di realizzazione del programma.

Gli sforzi furono coronati da successo, dal momento che alcune persone dell'Istituto di Storia dell'Università Adam Mickiewicz poterono tenere conferenze a Perugia. Tra di essi vi era anche l'ideatore dell'iniziativa da parte polacca. Il prof. Topolski incoraggiava altri docenti a tenere conferenze in italiano, poiché in tal modo cresceva fortemente l'interesse degli ascoltatori, e soprattutto degli studenti, ma non solo, poiché gli incontri avevano carattere di lezioni aperte. Occorre al contempo sottolineare che il prof. Topolski preparava in francese i resumé delle lezioni, che successivamente venivano tradotti fra l'altro in italiano ${ }^{7}$. Se si parla del successo della collaborazione con l'Università di Perugia vanno menzionate le ricerche del prof. Deodato sulla situazione economica della Libera Città di Danzica, con particolare considerazione per gli anni 1929-1932, mentre alto commissario della Lega delle Nazioni nella Libera Città era Manfredo Gravina ${ }^{8}$. Va aggiunto che la collaborazione avviata con Poznań e la Polonia dura fino ad oggi.

5 AUAM, Wyjazdy zagraniczne, nr 25, prof. Topolski, Sassari.

6 La nota bibliografica completa è la seguente: R. Righini, Między mitem a rzeczywistością: Indie w wyobrażeniach Rzymian od Oktawiana Augusta do II wieku [z jęz. wł. przeł. Jerzy Topolski], Poznań 1997, p. 19, (Xenia Posnaniensia: wykłady gościnne w Zakładzie Historii Społeczeństw Antycznych. Series Altera).

7 J. Topolski, Microstoria e macrostoria. Problemi metodologici, Perugia 1984-1985, pp. 15.

8 Testimonianza polacca di tale interesse è l'articolo sull'attività del Gravina, che utilizzava materiali precedentemente sconosciuti provenienti da collezioni private. Vd. E. Deodato, Manfredi Gravina - Wysoki Komisarz Ligi Narodów w Gdańsku (czerwiec 1929-wrzesień 1932), [in:] "Studia Historica Slavo-Germanica”, t. XV, 1986 (189), pp. $127-$ 153. Il testo era stato precedentemente pubblicato in Materiali di Storia 9. Annali della Facoltà di Scienze Politiche, a.a. $1983-1984$, pp. 32-66. 
Fra gli altri ricercatori italiani che hanno collaborato in modo amichevole con Jerzy Topolski e sua moglie Danuta si distinguono il professor Luciano Canfora (Bari), il professor Sebastiano Tafaro (Bari) - giurista, il professor Francesco Coniglione (Catania) - filosofo (questi era ospite di casa Topolski insieme al professor Jerzy M. Brzeziński), la professoressa Bianca Valota Cavallotti (Milano) - esperta di storia dell'Europa orientale e nipote del celebre storico romeno Nicolae Iorga, che fu a Poznań nel 1986 e nel 1992, Francesco Cataluccio della casa editrice Mondadori e, con speciali prerogative, il professor Silvio Zotta da Napoli e da Roma, del quale si farà ancora accenno.

Sì è detto sopra che la pubblicazione della Metodologia di Jerzy Topolski fu un avvenimento tenuto in considerazione non soltanto dagli storici italiani, ma anche dagli italianisti polacchi attivi nella Penisola. Il professor Sandro Schipani nel già menzionato invito all'Università di Sassari annotava la raccomandazione di Topolski fatta dal professor Bronisław Biliński, allora e per molti anni a venire direttore della filiale romana dell'Accademia Polacca delle Scienze, instancabile ed eccezionale propagatore dei contatti fra i due Paesi ${ }^{9}$. Non minor interesse accompagnò il libro del 1965, importante per la storiografia polacca: Narodziny kapitalizmu w Europie XIV-XVIII wieku. La traduzione italiana, apparsa a Torino nel 1979 con il titolo di La nascita del capitalismo in Europa. Crisi economica e accumulazione originaria fra XIV e XVII secolo contava 322 pagine. Varrebbe sicuramente la pena di prendere in esame le modifiche introdotte nella versione italiana, sia a causa della pubblicazione a quattordici anni di distanza dall'edizione polacca, sia in considerazione del lettore italiano. Una considerazione analoga potrebbe essere formulata per un altro titolo presente in ambito italofono dal 1981: La storiografia contemporanea. Nella versione originale il libro recava il titolo di Marksizm a historia [Il marxismo e la storia] ed era stato pubblicata nel 1977. Sarebbe interessante conoscere l'opinione del traduttore, Lionello Costantini ${ }^{10}$ sull'entità dei cambiamenti o degli adattamenti al mercato italiano, dove le ricerche sul marxismo

9 Non priva di valore in questa sede è una menzione degli enormi meriti del professor Bronisław Biliński (1913-1996) nello sviluppo della cosiddetta Accademia Polacca, che dopo la seconda guerra mondiale fu inclusa nell'Accademia Polacca delle Scienze con il nome di Sede Scientifica di Roma [Stacja Naukowa PAN w Rzymie]. Il prof. Biliński ne fu direttore per più di un quarto di secolo, fino al 1984. Di formazione filologo classico, divenne studioso delle relazioni polacco-italiane in varie epoche e settori. Il centesimo numero della serie Conferenze, pubblicate in italiano dall'Ossolineum, fu il suo opus magnum, che riassumeva l'attività a Roma e in tutta Italia. B. Biliński, Figure e momenti polacchi a Roma. Strenna di commiato, Wrocław-Warszawa-Kraków 1992, 504 pp. Donandomi un esemplare del libro in occasione di uno degli incontri presso l'Istituto di Cultura Italiana a Varsavia disse con malinconia: "Non c'è qui persona più degna di Lei che possa far buon uso di questo libro". Una recensione positiva è stata scritta da Tadeusz Bieńkowski in: "Kwartalnik Historii Nauki i Techniki", 1992, R. 37, nr 4, pp. 188-191.

10 Ciò non è possibile, poiché Costantini è morto nel 1994. Questi è stato un noto slavista italiano, professore all’Università La Sapienza di Roma, redattore di "Ricerche slavistiche", collaboratore dell'Istituto dell'Enciclopedia Italiana, esperto di storia e lingua serba, ma anche - soprattutto negli ultimi anni di vita - stimato traduttore. 
erano ancora trattate con la serietà consona a tale filosofia $a^{11}$. Tale considerazione è tanto più interessante se si considera che nel 1980 Jerzy Topolski visitò alcuni centri universitari - Firenze, Bari e Roma - fra cui l'Istituto Gramsci, diretto dal famoso storico italiano Paolo Spriano, autore di fondamentali lavori dedicati al ruolo della sinistra nel processo che condusse al cosiddetto compromesso storico.

Coronamento della presenza di Jerzy Topolski sul mercato editoriale italiano fu la monografia Narrare la storia: nuovi principi di metodologia storica. Nel frontespizio emerge che Raffaello Righini (1925-2001) fu coautore ("con la collaborazione"). Si trattava di un contatto importante, senza dubbio amichevole. Professore dell'Università di Torino e storico dell'antichità romana ${ }^{12}$, Righini fu organizzatore di un soggiorno di due mesi, l'unico di una certa durata, di Jerzy Topolski in Italia. Occorre aggiungere che il prof. Righini, che nel libro menzionato ha svolto la funzione di "collaboratore", ha lasciato soltanto alcuni brevi lavori. Fra questi l'opera realizzata in comune con Jerzy Topolski appare come il capolavoro di una vita. Vale la pena di esaminare la questione più da vicino. Questo lavoro - leggiamo nell'introduzione firmata dal prof. Topolski - non sarebbe venuta alla luce senza l'ispirazione del Righini, che ha sfruttato il mio soggiorno a Torino come visiting professor a cavallo del 1985/1986. Righini - informava poi Jerzy Topolski - ha offerto un grande contributo nella preparazione e alla redazione del testo italiano e ha avuto una grande influenza sul risultato finale.

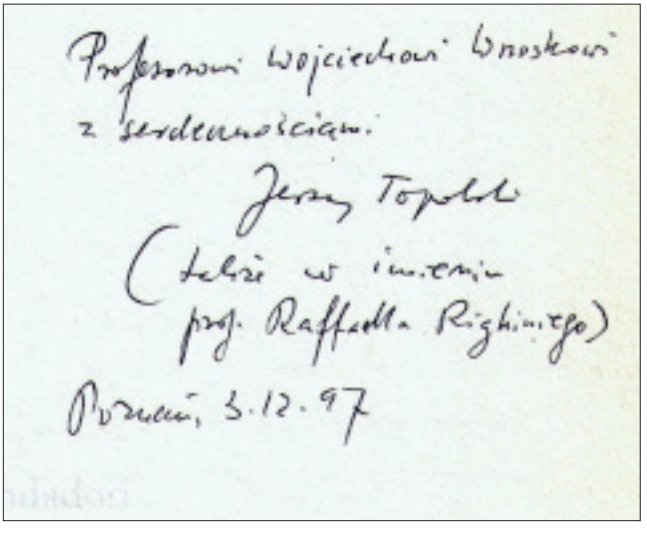

Danuta Eabędzka Topolska sottolinea ampiamente il ruolo svolto dal professor Raffaello Righini nella vita di tutta la famiglia: „Negli anni Novanta fu il più caro amico di Jerzy. Conducevano spesso lunghe conversazioni telefoniche su temi sia scientifici, sia personali. Si vedevano regolarmente in Italia. D'estate Topolski trascorreva lunghe vacanze con la figlia Ania ad Arbia, nella villa della

11 Per esempio Raffaele Righini, (In memoria. Jerzy Topolski, s.194) ha osservato che nell'edizione italiana è stato tralasciato il testo dedicato al leninismo in storiografia, pubblicato in alcune versioni nel 1970. Vd. la compilazione di Maria Danuta Topolska [in:] Jerzy Topolski - o nowy kształt nauki historycznej, red. M. Drozdowski, Warszawa-Poznań 1988, poz. 224, 232, 233, 256, 258, 266, 314, 397.

12 Il professor Wojciech Wrzosek lo definisce conoscitore del sanscrito. Vd. W. Wrzosek, Wycinanki, Poznań 2008, p. 7. Il Righini sopra citato (In memoria. Jerzy Topolski, pp. 179-200) offriva poche informazioni a proposito dei reciproci contatti. 
famiglia Righini o viaggiando con Raffaello per il Paese. Risultato di questi incontri fu non solo la pubblicazione di Narrare la Storia, ma anche il fatto che Ania diede l'esame di maturità di italiano, lingua non presente nel programma d'insegnamento del liceo Marcinek. Anche Raffaello veniva in Polonia. A questo proposito potrebbe raccontare di più il prof. Wojciech Wrzosek. Vorrei aggiungere che il 10 aprile 1990 si svolse nell'aula dell'Accademia Musicale di Poznań un recital pianistico della figlia di Raffaello, Federica Righini. Dopo la morte di Jerzy ci telefonava ogni domenica sera. Aveva anche incominciato a studiare il polacco"13.

$\mathrm{Va}$ aggiunto che il libro non ebbe particolare successo. Non si è riusciti a trovare alcuna recensione oltre alla nota dell'editore. Mondadori, inserendo il lavoro nella serie "Sintesi", incoraggiava all'acquisto del libro al prezzo di 20 euro scrivendo: "Dopo due libri, divenuti dei classici della riflessione contemporanea sul sapere storico (Metodologia della ricerca storica, 1975 e Teoria del sapere storico, $1983^{14}$ ), Topolski propone una nuova teoria della storiografia mettendola in relazione a una filosofia della storia che tiene conto dell'evoluzione del pensiero contemporaneo (Derrida, Barthes, Foucault, Ricoeur, Eco). Il libro fa un'analisi accurata del racconto storico, delle sue strutture retoriche, dell'uso delle fonti e dei condizionamenti ideologici che intervengono nella ricerca e nella scrittura. Un'opera di discussione, approfondimento e studio, che mancava in Italia" (1997). Questi aspetti ritornano nel testo pubblicato da Patrizia Fazzi in Diacronie, Studi di Storia Contemporanea (2015) con l'eloquente e chiaro titolo di Narrare la storia: la lezione di Jerzy Topolski. Ognuno può leggere questo testo, disponibile su internet ${ }^{15}$. Alcuni titoli italiani di Jerzy Topolski, che fu anche mio Professore ed Amico, sono stati da me collocati sulla piattaforma della Biblioteca Elettronica della Grande Polonia (Wielkopolska Biblioteka Cyfrowa). La dottoressa Danuta Łabędzka Topolska non solo ha acconsentito, ma anche ha inserito alcune importanti integrazioni. Un valido aiuto nella raccolta delle pubblicazioni italiane disperse del Professore è stato offerto dalla professoressa Ewa Domańska e dal professor Wojciech Wrzosek.

13 Integrazioni gentilmente offerte al testo originario da parte della dottoressa Danuta Łabędzka-Topolska. Fra i contatti polacco-italiani che vedevano protagonista la famiglia Topolski, avevano un carattere speciale quelli con il professor Silvio Zotta, attivo a Napoli e Roma. Questi, dopo essere stato precedentemente in contatto con Jerzy Topolski, venne a Poznan per una conferenza nel 1986. L'anno successivo sposò la sorella della signora Danuta, che da allora mantenne contatti familiari con l'Italia.

14 L'editore si richiamava ad un lavoro non pubblicato in italiano: Teoria wiedzy historycznej [Teoria della conoscenza storica], Poznań 1983, 509 pp. Questo lavoro ha suscitato in Polonia grande interesse e una discussione degna di tale interesse. Vd. M. D. Topolska, [in:] Jerzy Topolski - o nowy ksztalt nauki historycznej, p. 117, poz. 654.

15 P. Fazzi, Narrare la storia: la lezione di Jerzy Topolski, Diacronie. Studi di Storia Contemporanea: Costruire. Rappresentazioni, relazioni, comunità, 29/06/2015, URL:< http://www.studistorici.com/2015/06/29/fazzi, numero_22/ > [ultimo accesso: 20 marzo 2017]. 
La morte del prof. Topolski è stata per me come un fulmine a ciel sereno. Gli avevo parlato per telefono il giorno precedente (domenica) e ci eravamo accordati per un incontro in ospedale il lunedì. È mancato prematuramente, pur essendo ancora in ottima forma intellettuale e fisica, creativo, molto aperto nei confronti del prossimo, straordinariamente amichevole e - ho la certezza di affermarlo - privo di nemici.

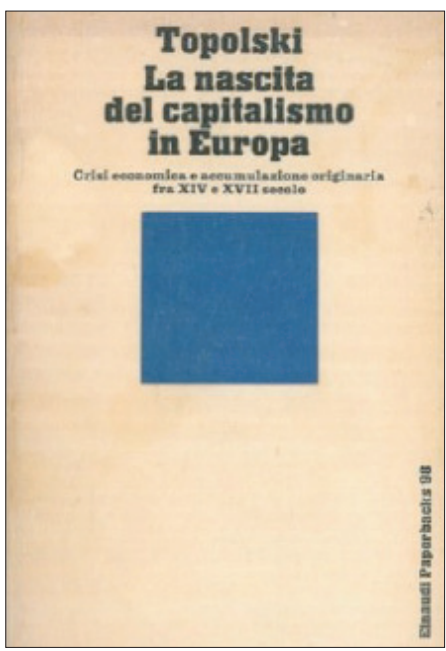

Fig 2 Dedica al Professor Alojzy Sajkowski

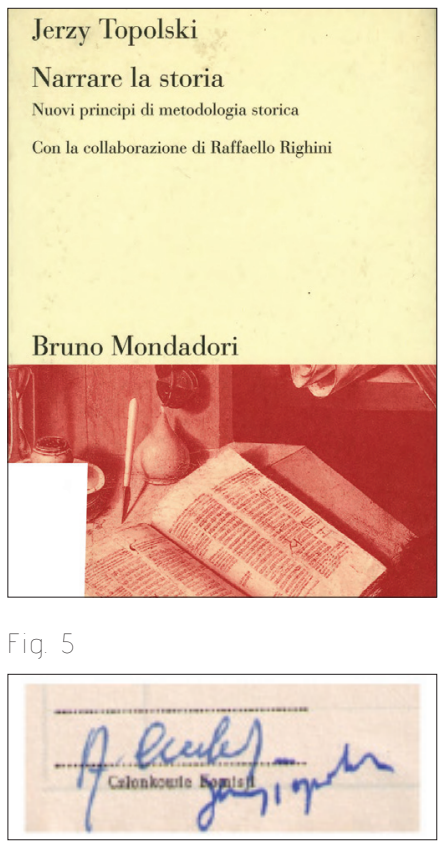

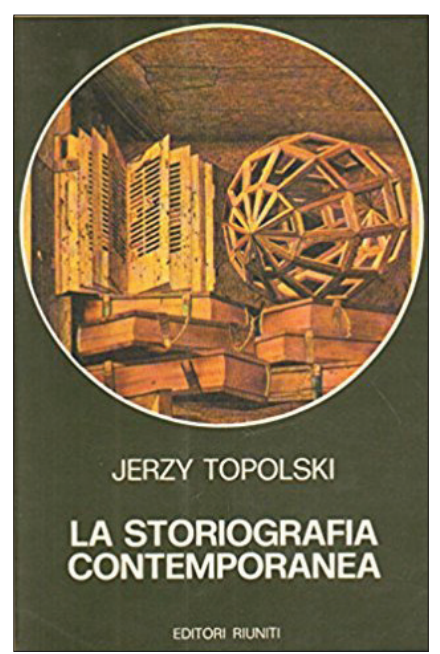

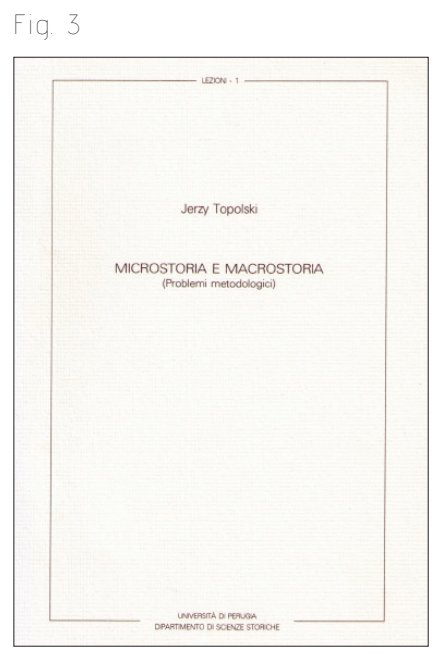

Fig. 6

Fig. 8 Nel mio libretto dopo la discussione della tesi di laurea magistrale

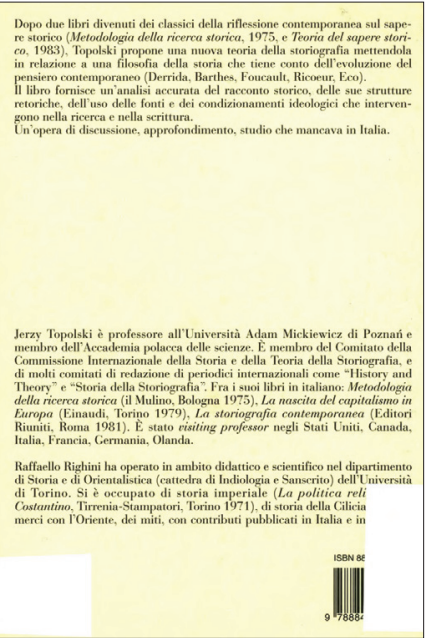

Fig. 4

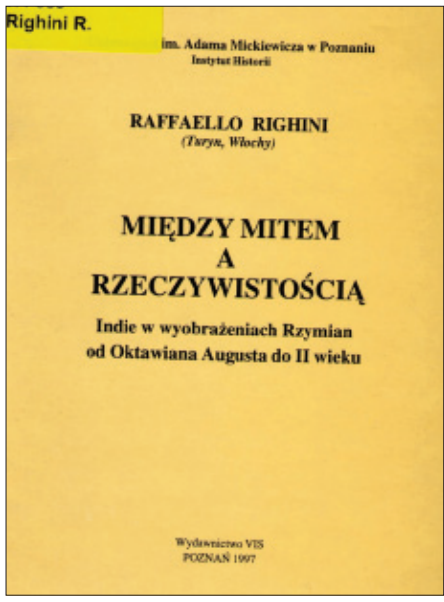

Fig. 7 